respectively. The rate of depressive symptoms was $42.8 \%$, and the average of well-being index in Korea was 58.3. The longer the working hours were, the higher the risk of depressive symptoms was. Even after adjusting for sex, age, socioeconomic position, physical problems, job stress and exposure to violence, the risks of depressive symptoms were significantly higher in people who worked 53-68 hours/week (PR, 1.13 95\% CI: 1.09 to 1.17 ), and more than 68 hours/week (PR, 1.07 95\% CI: 1.02 to 1.13 ) than 35-40 hours/week.

Conclusion We showed the long working hours could be an independent risk factor for depressive symptoms in employees. Long working hours can lead to the lack of time to recover and sleep disturbances, which can be associated with depressive symptoms. It is necessary to adjust to the appropriate working hours for employees' mental health.

\section{THE 2016 GLOBAL BURDEN OF DISEASE ARISING FROM OCCUPATIONAL EXPOSURES}

Tim Driscoll. Sydney School of Public Health, University Of Sydney, Sydney, Australia

\subsection{6/oemed-2018-ICOHabstracts.402}

Introduction There are a variety of hazards faced by workers across the globe. Many are common to workers in similar occupations or industries in different regions. Others comprehensive study of the occupational burden of disease has been most recently studied as part of the Global Burden of Disease (GBD) study, headed by the Institute of Health Metrics and Evaluation at the University of Washington.

Methods The risk factors section of the GBD study uses a population attributable fraction (PAF) approach, combining relative risk estimates from the published literature with estimates of exposure prevalence. The PAFs are applied to estimates of the total number of cases or Disability Adjusted Life Years (DALYs) of a particular disorder to produce estimates of the number of deaths or the overall burden (in DALYs) arising from individual occupational risk factors and groups of risk factors. The included risk factors are a range of carcinogens resulting in cancer; particulate matter, gases and fumes resulting in chronic obstructive lung disease; asthmagens causing asthma; noise causing noise-induced hearing loss; ergonomic risk factors causing low back pain; and injury risk factors resulting in injury.

Results The preliminary results for 2016 suggest that the largest burden in terms of deaths was from carcinogens, injury risk factors and particulate matter, gases and fumes. In terms of DALYs, the largest burden was from ergonomic factors, injury risk factors, particulate matter, gases and fumes and noise. The rates and PAFs varied by region and across age and gender.

Conclusion Studying the burden of disease arising from occupational exposures provides guidance to policy makers and practitioners regarding the scope of ill health resulting from work and where resources might most be needed.

\section{NEW EU-OSHA-APPROACH TO QUANTIFY EXPOSED WORKER POPULATIONS IN THE EU}

L Lieck*. European Agency for Safety and Health at Work, Bilbao, Spain

10.1136/oemed-2018-ICOHabstracts.403
Introduction The quantification of the number of workers that are exposed to dangerous substances is a key element for evidence based policy decisions, particularly in the area of occupational safety and health legislation and regulation of chemicals in general. In the past three decades a lot of scientific efforts were made to quantify the number of exposed workers at national, at EU and at international level. The methodological difficulties can be categorised in three major topics:

- The availability of reliable and representative exposure data

- The availability of substance and sector specific production and usage data

- The availability of detailed statistics on sectoral employment, occupations and major working tasks.

Methods The EU-OSHA approach is characterised by a combination of production and usage data from PRODCOM, SPIN and ECHA with survey data from the European Working Conditions Survey (EWCS), the Labour Force Survey (LFS) and the European Survey on New and Emerging Risks (ESENER). Moreover, these data are combined with structural business data of EUROSTAT. In a second step the findings are correlated with national datasets, e.g. from follow-up projects of CAREX or national cancer registers. Still the results of this correlation have to be justified by sector experts' assessment. Further datasets at European level (the planned EU OSH InfoSystem) or specific surveys on certain substances will improve the reliability and comprehensiveness.

Results Improved reliability and precision of quantitative assessment of the occurrence of exposure to substances in certain sectors and occupations, and the best possible estimate of the number workers exposed to these substances.

Discussion Public data on chemical substances is constantly increasing and providing more precision. It will be possible to update and improve the figures from previous estimation approach.

\section{ENVIRONMENTAL ASBESTOS EXPOSURE IN SOUTHERN ITALY: MESOTHELIOMA CASES DUE TO THE SAME POLLUTION SOURCE}

${ }^{1} \mathrm{~L}$ Vimercati*, 'D Cavone, 'L De Maria, ${ }^{1} \mathrm{~A}$ Caputi, , $\mathrm{M}$ Quarato, ${ }^{2} \mathrm{G}$ Serio. ${ }^{1}$ Interdisciplinary Department of Medicine, Occupational Medicine 'B.Ramazzini', University of Bari Medical School, Bari, Italy; ${ }^{2}$ Department of Emergency and Organ Transplantation (DETO), Pathology Division, University of Bari Medical School, Bari, Italy

\subsection{6/oemed-2018-ICOHabstracts.404}

Introduction Bari municipality (Apulia region, Southern Italy) has been defined as a contaminated site of national priority for remediation because of diffuse environmental contamination caused by an asbestos cement factory. Up to date, the regional mesothelioma registry listed 66 environmental mesothelioma cases among residents. We report two peculiar cases of malignant mesothelioma, one peritoneal and one pleural, due to residential asbestos exposure close to a military barracks, located inside the urban area and reclaimed only in 2006.

Methods The records of the two cases with environmental exposure were listed in the Apulia regional mesothelioma registry. The registry ensures complete, quality information on exposure and diagnosis thanks to the adoption of operative guidelines agreed at national level. Molecular analysis with CGH-array was performed on paraffin-embedded tissue. 
Results The peritoneal case in a 36-year-old man shows a peculiar characteristics long survival (more than 17 years). The patient was treated with cytoreductive surgery and hyperthermic intraperitoneal chemotherapy. Molecular analysis detected multiple chromosomal imbalances. The gains were prevalent. DNA copy number alterations (CNAs) observed loss at 1q21, 8 p 23.1 and gains at 3 p $22.2 \rightarrow 3$ p $22.1, \quad 3$ p $25.3 \rightarrow$ p 25.1 , $4 q 13.1$ and Xq22.2. These are novel CNAs here identified and uncommon in malignant peritoneal mesothelioma.

The pleural case, a 74-year-old man, suffering from a familial Becker type muscular dystrophy, opted for chemotherapy (alimta + cisplatinum) with external hyperthermia. At follow-up in April 2017, about 2 years from diagnosis and 21 months from the beginning of chemotherapy treatments, he has left shoulder pain, general weakness but good respiratory expansion.

Both cases lived in apartments overlooking military barracks from 1963 to 1999.

Discussion Past intensive use of asbestos has implied severe public health consequences among Bari inhabitants. Our study showed that the presence of the asbestos cement factory, as well as the military barracks, have been related with the onset of malignant mesothelioma among the neighbouring resident population.

\section{AVOIDANCE OF UNDER-REPORTING AND SELECTION BIAS IN OCCUPATIONAL INJURY AND ILLNESS SURVEILLANCE SYSTEM}

\footnotetext{
${ }^{1,2}$ Ping Hui Chen*, 1,2Pau Chung Chen. 'Department of Environmental and Occupational Medicine, National Taiwan University Hospital, Taipei, Taiwan; ${ }^{2}$ Institute of Occupational Medicine and Industrial Hygiene, National Taiwan University School of Public Health, Taipei, Taiwan
}

\subsection{6/oemed-2018-ICOHabstracts.405}

Introduction Under-reporting of occupational injury and illness (OII) and accompanied selection bias have always been a difficult challenge for policy making and epidemiological studies in many countries, and each country has come up with various reporting mechanisms to obtain reliable data from different sources. The object of this study is reviewing reporting mechanisms in nine countries, including Taiwan, Japan, Korea, Singapore, Australia, the United States, the United Kingdom, Germany, and France, and concluding a generalised model for constructing an ideal OII surveillance system.

Methods We carry out institutional comparison of various reporting mechanisms in different countries, both quantitatively and qualitatively, based on yearly statistics on OII from each reporting mechanism, and searchable online information, including statutory laws, official webpages, government documents and journal articles, respectively.

Results We categorise various reporting mechanisms into four types, including OSH-Act-based, compensation-based, medicalpractice-based, and health-examination-based, and all countries have more than two types of reporting mechanism. Each type has different reporting incentives, coming from fulfilling statutory reporting obligation by employer, claiming insurance benefit by employee, or seeking information feedback for clinical decision-making and clinical studies by physicians, and thus has its own unique strengths and weaknesses in reporting certain kinds of OII.

Discussion Due to different coverage of OII, each type of reporting mechanism has an irreplaceable role in OII surveillance. Thus, in addition to the operational effectiveness of individual reporting mechanism, emphasis should be put on inclusion of all four types of reporting mechanism with close coordination and integration in constructing an ideal OII surveillance system. To avoid under-reporting and accompanied selection bias, researchers should also get familiar with characteristics and OII coverage of certain reporting mechanism before the utility of reported statistics from that mechanism, and pooling data from more than one reporting mechanisms may be necessary for a more comprehensive picture of OII.

\section{PREVALENCE OF BACK PAIN IN PROFESSIONAL DRIVERS IN ABU DHABI}

Nahid Ebinouff, Omer Alnajeb. Ambulatary Health Services, Abu Dhabi, United Arb Emirates

\subsection{6/oemed-2018-ICOHabstracts.406}

\section{Aims}

1. The principle aim of the study is to examine whether being a driver will lead to an increase in the risk of back pain from the general population by ascertaining the prevalence of back pain among the professional drivers in Abu Dhabi (UAE) and to compare it to the prevalence of back pain in the general population.

2. To investigate whether the type of vehicle driven is a risk factor for back pain by comparing the prevalence of back pain among taxi drivers to the prevalence of back pain among bus drivers and heavy vehicle drivers.

Method It is a cross sectional study that look into the following:

- Prevalence of back pain among professional drivers in Abu Dhabi.

- Comparing the prevalence of back pain between taxi drivers, bus drivers and heavy vehicles.

- All professional drivers attend Al Madina Occupational Health Centre (Abu Dhabi) for all types of medical assessments (Pre-employment, periodic, fitness to return to work or fitness for the extension of services) who agree to participate are included in the study.

- The study ran from January 2015 up to May 2015.

- The sample size was 499.

- Data was collected by a self-filled questionnaire, which has been modified from a validated New Zealand acute low back pain questionnaire (permission taken).

The questionnaire includes general demographic data, work details and back pain information.

Result The study was carried out in Abu Dhabi, UAE. The study population was 499 (the response rate was 92\%). 27 questionnaires were excluded due to incomplete or missed information. The rest were 472 drivers, 141 of them disclose back pain in the previous 3 .

12 month, giving prevalence of $29.9 \%$. From those with back pain $92 \%$ deny any other health problems that affect their back. $82 \%$ of them described their pain as mild. $80.9 \%$ mentioned that minor physical activity does not make their pain worse. $87.2 \%$ declare that, they can do light work for an hour without pain being a problem.

In this study some occupational factors have been investigated with relation to back pain in professional drivers. It shows that, working hours $>8$ hours per day, working days $>5$ days per week and years of driving $>10$ years, are significantly associated with increased back pain in professional 